La Revue

des Droits

de l'Homme

\section{La Revue des droits de l'homme}

Revue du Centre de recherches et d'études sur les droits fondamentaux

6 | 2014

Revue des droits de l'homme $-\mathrm{N}^{\circ} 5$

\title{
Révolutions arabes et renouveau constitutionnel : une démocratisation inachevée
}

Malik Boumédiene

\section{OpenEdition}

\section{Journals}

Electronic version

URL: http://journals.openedition.org/revdh/959

DOI: $10.4000 /$ revdh.959

ISSN: 2264-119X

\section{Publisher}

Centre de recherches et d'études sur les droits fondamentaux

Electronic reference

Malik Boumédiene, «Révolutions arabes et renouveau constitutionnel : une démocratisation inachevée », La Revue des droits de l'homme [Online], 6 | 2014, Online since 18 November 2014 connection on 09 July 2020. URL : http://journals.openedition.org/revdh/959 ; DOI : https://doi.org/ 10.4000/revdh.959

This text was automatically generated on 9 July 2020.

Tous droits réservés 


\title{
Révolutions arabes et renouveau constitutionnel : une démocratisation inachevée
}

\author{
Malik Boumédiene
}

1 Le renouveau constitutionnel dans une partie du monde arabe trouve ses origines dans la conception et la pratique du pouvoir ainsi que dans la place restreinte jusqu'alors donnée aux droits fondamentaux. La question des droits de l'homme et de la démocratie dans le monde arabe "pré-révolutionnaire» faisait (et fait encore aujourd'hui) régulièrement l'objet d'une actualité.

2 Il n'y a qu'à prendre l'exemple de la Tunisie sous la présidence du président Ben Ali pour s'en convaincre. Ben Ali était au pouvoir depuis 1989. La démocratie politique était étouffée. Par ailleurs, sous sa présidence, les partis religieux étaient réduits au silence. Le RCD (Rassemblement constitutionnel démocratique- RCD) était le parti unique du Président Ben Ali créé par ses soins en 1988. Ce parti contrôlait, depuis sa création, la grande majorité des sièges du Parlement. L'Etat tunisien faisait l'objet de multiples critique du point de vue du respect des droits de l'homme. C'est ainsi que l'Etat s'est vu reconnaitre coupable par diverses ONG d'actes de torture ou de mauvais traitements dont certains magistrats avaient refusés d'enregistrer les plaintes. L'indépendance de l'autorité judiciaire demeurait fragile puisque le poids du pouvoir exécutif était important au sein du Conseil supérieur de la magistrature. La liberté d'association, et ainsi la place de la société civile, restait à conquérir. En effet, un nombre très limité d'associations indépendantes n'a été officiellement enregistré par les autorités et, en pratique, plusieurs associations de défense des droits de l'homme ont rencontré des obstacles dans l'obtention d'un tel enregistrement. On a également $\mathrm{pu}$ constater une certaine mise sous contrôle des citoyens à travers la décision du Président de la République le 10 mai 1991 de créer des comités de quartier. En janvier 1992, on comptait 2825 comités de quartier. En créant de tels comités, le pouvoir avait mis en place un dispositif nouveau visant à sa consolidation. Ils étaient contrôlés par des personnalités politiques (ministres, membres du RCD, Etat,...). 
3 Outre les aspects politiques, la question sociale est une question, également, omniprésente dans le monde arabe "pré révolutionnaire" (comme aujourd'hui encore). Au Maroc par exemple, le nombre de chômeurs tend à augmenter. Avec près de 1130000 de personnes sans emploi, le taux de chômage national a atteint, au premier trimestre de 2012, les $9,9 \%$, soit une hausse de 0,8 par rapport à 2011 (HautCommissariat au plan). Plus de $28,9 \%$ de la population vit sous le seuil de pauvreté (rapport du PNUD, 2010). Enfin, près de 53\% des marocaines ne savent ni lire, ni écrire, révèle un rapport du Haut-commissariat au plan marocain (2014).

4 La situation politique, économique et sociale d'une partie du monde arabe ne pouvait alors que constituer les bases à des "révolutions » futures. Ce qui fut le cas en 2011 avec la Tunisie à travers le départ du président Ben Ali. S'en suit un effet domino puisque avec le Maroc, l'Egypte, le Bahreïn, la Syrie ou encore la Libye. Ces «révolutions" ou «printemps arabes» vont se traduire par la mise en place de nouvelles constitutions. Ces dernières témoignent d'une volonté de démocratisation (I) même si cette dernière reste limitée, non aboutie, voire précaire (II).

\section{Le renouveau constitutionnel comme fondement à la démocratisation}

5 Il est incontestable que le monde arabe se voit traversé par un mouvement démocratique qui touche les fondements même des régimes politiques en place. Ce mouvement se manifeste par une volonté de consolider les droits fondamentaux (A) tout en recherchant un plus grand équilibre des pouvoirs (B).

\section{A. La consolidation des droits fondamentaux}

6 La place des droits fondamentaux, tout comme leur protection, au sein des constitutions devient aujourd'hui un indice pour se prononcer sur la densité démocratique d'un régime politique. A ce titre, le renouveau constitutionnel suite au "printemps arabe » est traversé par la volonté de donner une nouvelle portée aux droits de l'homme et, de manière générale, au droits fondamentaux tels que reconnus par le droit international. Prenons quelques exemples significatifs: le Maroc, la Mauritanie, la Syrie et l'Egypte.

\section{Le Maroc}

7 La consolidation des droits fondamentaux se matérialise à travers la nouvelle constitution marocaine en date du $1^{\mathrm{er}}$ juillet 2011. C'est ainsi qu'au sein du préambule il est dorénavant noté que le Maroc a pour objectif de construire un "Etat de droit démocratique ». Le préambule souligne aussi, explicitement, l'attachement du Royaume aux droits de l'homme "tels que universellement reconnus». En outre, l'article 9 fait bénéficier les partis politiques d'une certaine protection puisque ces derniers ${ }^{1}$, notamment, ne peuvent être suspendus ou dissous par les pouvoirs publics qu'en vertu d'une décision de justice.

8 L'égalité entre les hommes et les femmes fait l'objet d'une attention particulière puisque l'article 19 de la constitution dispose que l'homme et la femme jouissent à égalité des droits et libertés à caractère civil, politique, économique, social culturel ou 
environnemental. La loi fondamentale va même jusqu'à poser le principe de la nécessité d'une action positive de l'Etat afin de garantir ce principe d'égalité et, plus précisément, de parité entre homme et femme. Dans ce sens, il est créé au sein de la Constitution d'une Autorité pour la parité et la lutte contre toutes formes de discrimination. Ce principe d'égalité concerne aussi les fonctions électives où le législateur est invité à intervenir. Cette volonté de promouvoir les droits des femmes s'intègre dans le droit fil du droit international et plus précisément la convention des Nations-Unies sur l'élimination de toutes les formes de discrimination à l'égard des femmes en date du 18 décembre 1979.

9 Le droit à la vie est reconnu comme le premier des droits humain. L'article 23 de la Constitution de 2011 concerne la sureté de l'individu. C'est ainsi que la détention arbitraire ou secrète et la disparition forcée sont considérés comme des crimes. Des garanties sont consacrées également en ce qui concerne la procédure pénale puisque toute personne détenue doit être informée immédiatement d'une façon qui lui soit compréhensible des motifs de sa détention et de ses droits dont celui de garder le silence. Elle doit bénéficier au plus tôt d'une assistance juridique. L'article 23 reconnait également la présomption d'innocence.

Enfin, l'article 28 concernera lui la liberté de la presse qui ne peut être limitée par aucune forme de censure préalable.

\section{La Mauritanie}

11 Un mouvement comparable touche également la Mauritanie, comme en témoigne la réforme constitutionnelle du 20 mars 2012 qui va dans le sens d'une consolidation des droits fondamentaux. On a peu parlé de la Mauritanie lorsque l'on aborde la question du printemps arabe. Pourtant, en janvier 2011, une personne de 43 ans se disant "mécontent de la situation politique du pays et en colère contre le régime en place", s'est immolée par le feu devant le palais présidentiel. Cet acte est intervenu quelques jours après des actes similaires survenus en Tunisie, en Egypte et en Algérie. Il s'agissait pour cette personne de protester contre le régime du général Mohamed Ould Abdel Aziz arrivé au pouvoir par un coup d'Etat militaire mené en août 2008, puis ensuite élu à la présidence de la République en juillet 2009.

La contestation qui débute vers le 17 janvier 2011 est décrite comme la plus grande depuis l'indépendance du pays. Les manifestations demandent des réformes sociales et économiques ainsi que le départ du président Mohamed Ould Abdel Aziz.

13 Face à cette situation, le gouvernement a adopté différentes mesures : le 23 juin, la pénalisation des délits de presse est levée et des terres sont distribuées à une centaine de jeunes diplômés pour qu'ils les cultivent. Le 20 mars 2012, la Constitution est modifiée. Elle reconnait l'égalité entre homme et femme en ce qui concerne les mandats électoraux et les fonctions électives. L'esclavage est condamné : nul ne peut être réduit en esclavage ou à toute forme d'asservissement de l'être humain, ni soumis à la torture et autres traitements cruels, inhumains ou dégradants. Cette condamnation de la torture s'inscrit dans le sillage de la convention des Nations-Unies contre la torture en date du 10 décembre 1984. Ces pratiques constituent des crimes contre l'humanité et sont punis comme tels par la loi. En outre, une Commission Nationale des Droits de l'Homme est créée. Il s'agit d'une institution consultative indépendante de promotion et de protection des droits fondamentaux. 


\section{La Syrie} faire appel et de plaider devant un jury. En outre, pèse dorénavant sur l'Etat l'obligation de fournir une assistance juridique à ceux qui ne possèdent pas de moyens financiers suffisant afin d'assurer leur défense. D'autres dispositions, encore, sont relatives à la procédure pénale. Il est reconnu dans ce sens dorénavant que lors de son arrestation la personne arrêtée doit être informé de ses droits et des motifs de son arrestation. Il est fait interdiction de garder une personne sous le contrôle de l'autorité administrative si ce n'est en vertu d'un ordre de l'autorité judiciaire compétente. Il s'agit ici de condamner les détentions arbitraires contraires à l'article 9 du Pacte international du 16 décembre 1966 relatif aux droits civils et politiques. Enfin, les personnes se voient reconnaître un droit à indemnisation si le verdict est erroné et la peine appliquée.

\section{L'Egypte}

Pour sa part, la Constitution égyptienne de 2014 reconnait un certain nombre de droits fondamentaux nouveaux, vient en préciser d'autres ou encore en renforcer certains déjà existants.

18 A ce titre, on constate que le préambule de la constitution fait référence à la déclaration universelle des droits de l'homme, les universités doivent enseigner les droits de l'homme (article 24) et l'article 93 dispose nouvellement que «L'Etat s'engage à respecter les traités, accords et conventions internationales relatifs aux droits de l'homme ratifiés par l'Egypte».

On relèvera tout d'abord que le droit de propriété bénéficie d'une attention particulière puisqu'il est non seulement garantie mais également «protégé» et que la liberté de culte devient « absolue».

20 A la lecture de plusieurs dispositions, le droit à la sûreté semble également consolidé. Par exemple, il est mentionné que toute personne arrêtée ou détenue doit être informée «immédiatement » des motifs par écrit (on voit là l'application de l'article $9 \$ 2$ du Pacte de 1966 relatif aux droits civils et politiques). Elle ne peut, en outre, être interrogée qu'en présence d'un avocat qui doit être désigné en cas de besoin (article 54). De plus, toute personne arrêtée ou détenue a le droit de s'en plaindre devant le tribunal qui devra alors trancher au bout d'une semaine. Dans le cas contraire, elle doit être remise immédiatement en liberté. Le nouveau texte reprend l'idée de l'inviolabilité $\mathrm{du}$ domicile mais vient encadrer les atteintes éventuelles à ce principe. C'est ainsi que 
s'il est permis d'entrer au domicile d'une personne, de le perquisitionner, ou de le surveiller ce n'est que dans les conditions déterminées par la loi et sur ordonnance judiciaire motivée, précisant le lieu, la date et l'objectif de la perquisition. En outre, les habitants de la maison doivent être prévenus avant toute perquisition.

En ce qui concerne la liberté de la presse, la nouvelle Constitution met l'accent sur le fait que les journaux. En outre, le texte fondamental mentionne qu'il est interdit de censurer, confisquer suspendre ou fermer de quelques manière que ce soit les journaux et médias égyptiens. Les journaux peuvent être publiés dès leur déclaration. La liberté de réunion est davantage protégée par rapport au texte de 1971 puisqu'il est indiqué qu'il est, non seulement, interdit aux agents de sécurité de participer à ses réunion mais, également, il est fait interdiction de mettre sur écoute celles-ci.

L'interdiction de la mise sur écoute ( espionner ») apparaît comme le nouvel apport du nouveau texte fondamental. Les syndicats bénéficient d'une protection renforcée dans la mesure où leur dissolution ne peut intervenir, dans l'avenir, que sur la base d'une décision judiciaire allant dans le sens de la philosophe de la Convention $n^{\circ} 87$ de l'OIT en date de 1948 qui est relative à la liberté syndicale et à la protection du droit syndical. Par ailleurs, le droit de grève est reconnu.

Les droits sociaux bénéficient d'une certaine attention de la part du texte fondamental puisque l'Etat doit assurer les services de l'assurance sociale. Il est indiqué que tout citoyen a le droit à la solidarité sociale, s'il est incapable de prendre en charge sa propre personne, ni sa famille, ou en cas d'incapacité, de chômage, de vieillesse, afin de leur assurer un minimum de moyen d'existence. De nouvelles dispositions viennent également mettre à la charge de l'Etat l'obligation d'assurer une pension convenable aux petits paysans, aux ouvriers agricoles, à la main d'œuvre saisonnière et à tous ceux qui ne sont pas couvert par le système de l'assurance sociale. La référence au droit au logement apparait aussi comme une nouveauté. Dans ce sens le droit à une habitation convenable est reconnu. A cette fin l'Etat doit adopter un plan national pour le logement basé sur la justice sociale, et qui encourage les initiatives individuelles et les coopératives de l'habitat. Ces dispositions sociales s'intègrent dans le sillage des dispositions de la déclaration universelle des droits de l'homme en date de 1948.

On remarquera enfin que la protection de l'enfance fait l'objet d'une attention particulière, contrairement au texte constitutionnel de 1971. Il est fait ainsi interdiction d'embaucher un enfant avant l'âge de la fin de l'enseignement primaire ou dans des activités inappropriées à son âge. En matière judiciaire, l'enfant ne peut être détenu ou déclaré responsable que dans les conditions très strictes de la loi. Une assistance juridique doit lui être assurée. Le lieu de détention doit être convenable mettant en place une séparation entre les sexes, l'âge, la nature du crime et l'éloignement des lieux de détention des adultes. Enfin, de manière générale, l'article 71 de la loi fondamental souligne que l'Etat doit prendre soin des enfants et des jeunes, assurer leur éducation, leur développement spirituel, moral, culturel, scientifique, physique, psychologique, social et économique tout en œuvrant pour leur participation politique. On le voit, ces nouveaux droits à destination des enfants trouvent un fondement dans la Convention des droits de l'enfant en date de 1989. 


\section{B. La recherche de l'équilibre des pouvoirs}

postrévolutionnaires. Il s'agit de la volonté du constituant de rechercher un certain équilibre des pouvoirs. L'équilibre des pouvoirs doit permettre un contrôle réciproque d'un pouvoir sur un autre et de constituer une garantie à la concentration du pouvoir au sein de l'exécutif ou du pouvoir législatif. Comme le précisait Montesquieu «Pour qu'on ne puisse abuser du pouvoir, il faut que par la disposition des choses, le pouvoir arrête le pouvoir $~^{2}$. Les réformes constitutionnelles ${ }^{3}$ égyptienne, mauritanienne, marocaine ou encore syrienne témoignent de ce mouvement constitutionnel.

\section{L'Egypte}

26 En Egypte, nous assistons, avec la réforme de 2014, à un affaiblissement du pouvoir présidentiel. On observe une réduction et un encadrement des prérogatives présidentielles qui se matérialise de différentes façons.

27 Encadrement : en témoignent les dispositions relatives au droit de dissolution. C'est ainsi que, dorénavant, le Président de la République ne peut dissoudre la Chambre des députés qu'après un référendum et sur la base d'un décret motivé. En outre, on remarque que le mandat du chef de l'Etat se voit réduit passant de 6 à 4 ans et celui-ci ne peut se représenter dorénavant qu'une seule fois. La déclaration de l'Etat d'urgence par le chef de l'Etat est également davantage encadrée puisque sous, le régime de la Constitution de 1971, la déclaration de l'état d'urgence devait être soumise dans les 15 jours à l'assemblée législative, ce délai est ramené à 7 jours.

28 Cet affaiblissement de la fonction présidentielle se manifeste non seulement par un plus grand encadrement des prérogatives du chef de l'Etat mais aussi par la suppression de certaines prérogatives. En ce sens, la Constitution de 2014 supprime les dispositions telles que celles contenues au sein de l'article 74 de la Constitution de 1971 et qui concernaient les pouvoirs exceptionnels du chef de l'Etat. Cet article disposait que : «En cas de danger imminent et grave menaçant l'unité national, ou la sécurité de la patrie, ou empêchant les institutions de l'Etat de remplir leur rôle constitutionnel, il appartient au président de la République de prendre les mesures urgentes pour parer à ce danger après consultation du Premier ministre et des présidents de l'assemblée du Peuple et de 'Assemblée consultative. Il adresse un message au peuple et fait procéder à un référendum sur les mesures prises dans les 60 jours qui suivent. La dissolution de l'Assemblée du peuple et de l'Assemblée consultative est interdite lors de l'exercice de ces pouvoirs ".

29 La réduction des prérogatives présidentielles se matérialise enfin par le fait que la nouvelle Constitution semble imposer au Président de la République de choisir le Premier ministre au sein du parti détenant la majorité des sièges à la Chambre des députés. Il s'agit là de donner une pleine possibilité pour le gouvernement de pouvoir prendre les grandes décisions en s'appuyant sur une majorité parlementaire. Il s'agit aussi d'encadrer le pouvoir de nomination du Chef de l'Etat et d'assoir un véritable régime parlementaire.

30 A côté de cette volonté de mieux encadrer les prérogatives présidentielles, il existe aussi celle de renforcer la place du pouvoir législatif afin de tendre vers un meilleur équilibre des pouvoirs. La structuration même de la constitution permet d'avancer une 
telle idée puisque la section relative au pouvoir législatif précède celle qui concerne le chef de l'Etat contrairement à la constitution de 1971.

31 C'est ainsi que la chambre des représentant voit le nombre de ses élus composé d'un minium d'élu qui est de 450 contre 350 sous la constitution de 1971. La convocation de la Chambre des représentants en sessions extraordinaire est facilitée puisque dorénavant elle peut être convoquée par au moins un dixième des membres (article 116) alors que sous le régime de la constitution de 1971 une motion devait être adoptée par la « majorité » de l'assemblée. En outre, on peut observer que le Premier ministre, comme ses ministres, doit répondre "obligatoirement » aux questions posées par un membre de la chambre des représentants et cela dans "la même session». Les membres de la chambre peuvent aussi interpeller le Premier ministre, les ministres et leurs adjoints et le débat sur l'interpellation doit intervenir dans les 60 jours de son dépôt. L'article 134 permet à chaque membre de la chambre de demander une réunion d'urgence ou une déclaration du Premier ministre, à l'un de ses adjoints, à un des ministres ou de leurs adjoints, sur des questions urgentes touchant à l'intérêt public. En outre la constitution nouvellement rédigée impose au Premier ministre comme aux ministres d'être obligatoirement présents à la Chambre si celle-ci le demande.

\section{Le Maroc}

Tout comme la nouvelle Constitution Egyptienne il semble que la réforme constitutionnelle marocaine de 2011 est fondée aussi sur la volonté de tendre vers davantage d'équilibre des pouvoir. Un tel fondement est inscrit dans la Constitution et plus précisément au sein de l'article $1^{\mathrm{er}}$.

Cet équilibre des pouvoirs va se manifester, dans un premier temps, par un encadrement du pouvoir royal. Le Roi à obligation dorénavant de nommer le chef du gouvernement au sein du parti politique arrivé en tête des élections des membres de la chambre des représentants.

La recherche de l'équilibre des pouvoir se matérialise aussi à travers la nouvelle Constitution à travers la volonté de renforcer le rôle du parlement. Le nouveau texte précis ainsi que le parlement contrôle l'action du gouvernement et évalue les politiques publiques. L'article 71 élargit le domaine de la loi qui pourra intervenir dorénavant, par exemple, dans le domaine des libertés et des droits fondamentaux.

Le contrôle du pouvoir législatif sur l'exécutif se manifeste aussi à travers l'article 102 qui permet aux commissions compétentes dans chacune des deux chambres de demander à auditionner les responsables des administrations. Cet équilibre des pouvoirs se manifeste enfin à travers le droit qui est donné dorénavant au chef du gouvernement de dissoudre la chambre des représentants sur la base d'un décret pris en conseil des ministres.

\section{La Syrie}

Un dernier exemple constitutionnel tend à appuyer l'idée de la volonté de donner une certaine réalité au principe d'équilibre des pouvoirs dans ce mouvement de renouveau constitutionnel touchant le monde arabe. Il s'agit de la révision constitutionnelle syrienne du 26 février 2012. 
chef de l'Etat pouvait exercer le pouvoir législatif en dehors des sessions de l'Assemblée. La constitution de 2012 vient préciser qu'il exerce un tel pouvoir en cas de dissolution de l'Assemblée du peuple uniquement. Ensuite si les prérogatives du chef de l'Etat ont été réduites celles-ci font l'objet dorénavant d'un encadrement plus poussé. En effet, la constitution du 26 février 2012 vient indiquer explicitement, dans son article 96, que le chef de l'Etat doit respecter la constitution. Il ne peut effectuer également que deux mandat successifs. L'élection présidentielle n'est plus au-dessus de la loi puisqu'une disposition dispose qu'une Haute Cour constitutionnelle est compétente pour examiner les recours concernant l'élection présidentielle. Enfin, le déclenchement de l'état d'urgence fait l'objet d'un encadrement contrairement au régime constitutionnel qui dominait sous la constitution de 1973 puisque dans l'avenir s'il revient au chef de l'Etat de décréter l'état d'urgence c'est seulement à la suite d'une décision du conseil des ministres adoptée à la majorité des $2 / 3$. En outre, ces décisions doivent être soumises à l'Assemblée du peuple dès sa première séance.

\section{La démocratisation à l'épreuve du renouveau constitutionnel}

Ce renouveau constitutionnel ne peut faire l'économie de certaines critiques. Il semble que l'on peut avancer d'une part, que la protection des droits fondamentaux restent lacunaire (A) et, d'autre part, que la place de l'exécutif et, plus précisément des chefs de l'Etat, reste prépondérante (B).

\section{A. Une protection fragile des droits fondamentaux}

41 La protection des droits fondamentaux, si elle fait l'objet d'une attention particulière au sein des constitutions postrévolutionnaires, mérite néanmoins d'être relativisée dans la mesure où plusieurs limites textuelles (1) et pratiques (2) apparaissent. 


\section{Arguments de texte}

\section{a) Le cas de l'Egypte}

A propos de l'Egypte, tout d'abord, on peut s'étonner que le texte fondamental précise que les principes de la Charia Islamique sont la source principale de la législation. Quid alors de la mise en place de droits fondamentaux en direction des femmes par exemple? Par ailleurs, en ce qui concerne la protection de la liberté religieuse, il est reconnu la construction de lieu de cultes uniquement pour les relions « révélées ». Quid des autres religions ou mouvements philosophiques? De même, l'article 72 indique que l'Etat doit garantir la neutralité des médias dont il est propriétaire. Mais quelle est l'effectivité de telles dispositions ? On peut en douter lorsque l'on regarde le traitement médiatique dont bénéficie le Président EL SISI. Ou encore, la constitution interdit la constitution de partis politiques sur une base religieuse. Il s'agit là d'une atteinte aux libertés politiques. La liberté syndicale se voit réduite puisque il est mentionné qu'aucune profession ne peut créer plus d'un syndicat et ces derniers ne peuvent exister dans les institutions étatiques. En outre, le droit de pétition est interdit puisque l'article 85 dispose « Aucune requête ne peut être faite au nom d'un groupe ». S'il existe des dispositions concernant le droit de l'environnement celles-ci ne font aucune référence, par exemple, aux principes de précaution, de participation ou encore de responsabilité environnementale. Concernant le procès pénal certains grands principes sont reconnus comme la présomption d'innocence ou le double degré de juridiction. Néanmoins on peut regretter l'absence de référence à la notion de droit à réparation ou encore du droit de voir sa cause entendu dans un délai raisonnable comme le souligne l'article 14 du Pacte international relatif aux droits civils et politiques (16 décembre 1966). Egalement, est reconnue la liberté de croyance. Il aurait été toutefois opportun de mentionner que nul ne peut être inquiété pour ses opinions religieuses à l'instar de l'article 10 de la Déclaration des droits de l'homme et du citoyen de 1789.

Plus généralement, il est fait référence à la notion de liberté mais aucune définition n'est proposée, pas même comme à l'article 4 de la Déclaration des droits de l'homme et du citoyen, que «La liberté consiste à pouvoir faire tout ce qui ne nuit pas à autrui ». Surtout, les droits et libertés reconnus par la loi fondamentale reste conditionnés par leur mise en œuvre par le législateur et donc de l'existence d'une volonté politique. D'autre part, la mise en œuvre de droits tels que le droit au logement, le droit à la santé ou le droit à l'éducation sont des droits qui ont un coût financier important. Or, quid de la capacité financière de l'Egypte qui apparait comme un pays pauvre dont le PIB est de seulement 225,9 milliards de dollars (2011)?

Enfin, concernant la protection des droits fondamentaux le texte constitutionnel met en place un contrôle a priori de la loi. Néanmoins il aurait été, là également, opportun d'aller plus loin en mettant en place la possibilité pour le justiciable de saisir la Cour constitutionnelle au cours d'un procès, c'est-à-dire la reconnaissance d'un contrôle $a$ posteriori de la loi. Un tel contrôle existe, notamment, au sein de la Constitution du Tchad, en date du 14 avril 1996, qui dispose, dans son article 166, que " Tout citoyen peut soulever l'exception d'inconstitutionnalité devant une juridiction dans une affaire qui la concerne. Dans ce cas, la juridiction sursoit à statuer et saisit le Conseil constitutionnel qui doit prendre une décision dans un délai de quarante-cinq jours ". 


\section{b) Autres exemples} référence aux discriminations entre hommes et femmes. En outre, la liberté de religion garantie par l'article 14 trouve sa portée limitée puisque l'Etat ne protégera pas une religion si celle-ci est contraire à l'ordre public ou encore aux bonnes mœurs. Qu'entend-on alors par ordre public et bonnes mœurs ?! Il s'agit là de deux expressions qui laissent une certaine marge de manœuvre aux pouvoirs publics. On remarque également qu'il n'est pas fait référence à la question des droits de l'homme ou à un texte international sur ce sujet. Par ailleurs, le droit à la protection sociale, à la retraite ou encore à la santé fait défaut alors que ces droits sont reconnus par les différentes conventions élaborer dans le cadre de l'O.I.T. Aucune disposition ne concerne la procédure judiciaire : présomption d'innocence, droit à un avocat ; droit d'accéder à un tribunal indépendant ; droit d'être jugé dans un délai raisonnable ; droit de faire appel ; droit à réparation en cas d'erreur judiciaire. Pourtant la Jordanie a ratifié le 28 mai 1975 le pacte du 16 décembre 1966 relatif aux droits civils et politiques qui reconnait dans son article 14 un certain nombre de droits à la défense au cours du procès pénal.

En Mauritanie, les droits sociaux sont exclus de la Constitution. Or, dans la grande majorité des constitutions on retrouve le droit à la sécurité sociale, le droit au logement, le droit à la retraite, la protection de la santé. Aucune disposition ne concerne le droit d'asile. Le principe de la démocratie ne figure pas au sein du préambule. Il n'est pas fait référence à la liberté de religion. Il n'est fait mention à la notion de droits de l'homme ou encore à une convention internationale à ce sujet que dans le préambule et non au sein des articles mêmes de la Constitution.

En Syrie, s'il est fait référence à la notion de droits de l'homme, aucun lien n'est fait avec le droit international des droits de l'homme. En outre, alors que, dans une démocratie, l'opposition joue un rôle fondamental - en ce sens que la Constitution marocaine consacre à l'opposition un statut particulier et protecteur -, rien de tout cela n'est prévu par la Constitution Syrienne. Enfin, le droit à la sécurité sociale est reconnu vis-à-vis des travailleurs, mais quid des autres catégories de la population?

$\mathrm{Au}$ Maroc pour finir, la Constitution vient limiter l'action des partis politiques et plus précisément la liberté d'expression. Ces derniers ont interdiction de porter atteinte à la religion musulmane ou encore à la forme monarchique du régime politique (article $7 \mathrm{de}$ la Constitution). On observe également que la religion musulmane bénéficie d'une place particulière au sein de la Constitution puisque on peut lire, au sein du préambule que « La prééminence » est accordée à la religion musulmane. Quid alors de la place et de la protection accordée aux autres religions?!

\section{La persistance de pratiques contraires aux droits et libertés}

50 Les limites juridiques à la protection des droits fondamentaux au sein des constitutions postrévolutionnaires ne sont pas les seules. La pratique du pouvoir, comme la législation en vigueur, peuvent aussi venir contredire les principes reconnus par les textes fondamentaux. Nous prendrons les exemples du Maroc et de la Syrie 


\section{a) Le Maroc}

51 On a pu constater, en 2012, qu'un tribunal marocain de la ville de Taza a condamné Abdelsamad Haydour, un étudiant de 24 ans, à 3 ans de prison et 1200 USD (environ 900 euros) d'amende pour avoir critiqué le roi du Maroc dans une vidéo publiée sur YouTube. M. Haydour a alors été accusé "d'avoir attenté aux valeurs sacrées de la nation ». Or, celui-ci n'a bénéficié d'aucune assistance juridique lors de l'audition et le tribunal n'a pas désigné d'avocat pour le défendre comme le requiert pourtant la législation du pays. Pourtant l'article 25 de la constitution reconnait la liberté de pensée, d'opinion, et d'expression sous toutes ses formes.

La liberté d'association dans la pratique reste également un droit à conquérir comme en témoignent les évènements d'aout 2011. Le samedi 20 août 2011, une centaine de juges s'était déplacé de différentes régions du Maroc pour participer à l'Assemblée générale constitutive du "Club des juges du Maroc ", à l'Ecole nationale de l'industrie minérale (ENIM) de Rabat. Cette organisation " veut cuuvrer pour garantir les libertés des citoyens à travers l'indépendance de la justice ". Cependant, l'adjoint du directeur de l'ENIM, interdit aux magistrats l'accès à l'école. Celui-ci avait reçu des instructions orales du ministère de l'Intérieur interdisant l'accès aux locaux qui pourtant ont été loués. Les magistrats se sont alors réunis dans la rue. Cette décision est contraire à l'article 111 de la Constitution marocaine qui garantit le droit pour les magistrats d'adhérer et de créer une association professionnelle.

53 A côté de la liberté d'association, la liberté de manifestation est aussi quelques fois bafouée. C'est ainsi qu'au mois d'aout 2013 les forces de l'ordre ont réprimé des manifestations Alors même que l'article 29 de la constitution dispose que "Chacun a le droit d'organiser des réunions et des manifestations pacifiques et non armées sans autorisation préalable mais avec déclaration préalable pour les manifestations dans un lieu public ».

\section{b) La Syrie}

54 Des pratiques très problématiques peuvent également être observées en Syrie. Dans un rapport de septembre 2012 une commission internationale d'enquête fait état de meurtres, d'exécutions sommaires, d'actes de torture, d'arrestations arbitraires, de violences sexuelles, de violations des droits de l'enfant de la part des forces gouvernementales. Se pratique également en Syrie de la part du gouvernement à des arrestations arbitraires. Par exemple Amnesty international a pu préciser que le militant politique Ali Sayed al Shihabi, réfugié palestinien vivant en Syrie, a été arrêté par les autorités syriennes le 19 ou le 20 décembre 2012 et soumis à une disparition forcée. Il faut noter que la Syrie est depuis 1963 sous le régime de l'état d'urgence permettant de réduire les libertés publiques.

Par ailleurs, l'égalité des sexes est reconnue par la Constitution syrienne. Pourtant les lois relatives au statut personnel et le code pénal contiennent des dispositions discriminatoires à l'égard des femmes et des filles, notamment en matière de mariage, de divorce, de garde des enfants et d'héritage. En outre, on note que si le code pénal n'exonère plus totalement les auteurs de prétendus crimes d'honneur, il laisse encore aux juges une certaine latitude pour infliger des peines réduites si un crime a été commis avec une intention "honorable». Enfin, la loi sur la nationalité de 1969 interdit aux femmes syriennes ayant épousé un étranger la possibilité de transmettre leur nationalité à leurs enfants ou à leur conjoint. 


\section{B. La prédominance des chefs de l'Etat} pouvoir exécutif et, plus particulièrement, du chef de l'Etat semble perdurer. Cette tendance pourrait trouver un fondement dans la survivance d'un schéma constitutionnel antérieur où la loi fondamentale consacrait de véritables régimes autoritaires. Prenons l'exemple de la Syrie, du Maroc, de l'Egypte, de la Jordanie et de la Mauritanie.

\section{La Syrie}

57 Il ressort de la lecture de la Constitution syrienne que le président de la République conserve de larges prérogatives. Il lui revient ainsi de nommer le premier ministre, les ministres et leurs adjoints et de les décharger éventuellement de leurs fonctions. C'est à lui qu'il appartient de déterminer la politique de l'Etat en accord avec le conseil des ministres qu'il lui revient de présider. Il peut demander des rapports tant au Premier ministre qu'aux divers ministres.

ssède un rôle au niveau international puisqu'il accrédite les chefs des missions diplomatiques auprès des gouvernements étrangers et il reçoit les accréditations des chefs de missions étrangères en Syrie. Il ratifie les traités et accord internationaux. Au niveau militaire, il est le chef suprême de l'Armée et de toutes les forces armées. Il prend toutes les décisions nécessaires à l'exercice de cette autorité.

Son influence est réelle sur l'Assemblée nationale dans la mesure où il peut s'adresser à celle-ci par des messages écrits et, en personne, faire des déclarations devant elle. Il a la possibilité également de dissoudre une telle assemblée. Il peut préparer des projets de loi et les transmettre au pouvoir législatif pour délibération. L'article 113 lui permet même d'exercer le pouvoir législatif, le cas échéant, en dehors des sessions de l'Assemblée populaire, en cas de dissolution de l'Assemblée du peuple.

On constate, à côté de ses prérogatives à l'égard du pouvoir législatif, que le président de la République possède des pouvoirs exceptionnels dans certains cas. Par exemple, si un grand danger menace l'unité nationale, la sécurité ou l'indépendance de la patrie ou empêche les institutions de l'État de remplir leurs obligations constitutionnelles, il peut prendre les mesures d'urgence exigées par les circonstances, en vue de faire disparaitre ce danger. Il a la possibilité de s'affranchir de la représentation nationale en faisant appel directement au peuple à travers le referendum sur les questions importantes relatives aux intérêts supérieurs du pays.

61 Il nomme par décret les membres de la Haute Cour constitutionnelle ce qui conduit à s'interroger sur l'indépendance et l'impartialité de cet organe. Enfin, il dispose de l'initiative de la révision, pouvoir partagé avec l'Assemblée du Peuple.

\section{Le Maroc}

62 Au Maroc, le Roi conserve de larges prérogatives malgré la réforme du $1^{\mathrm{er}}$ juillet 2011. Il a, par exemple, la possibilité de mettre fin aux fonctions d'un ou plusieurs membres du gouvernement. Il peut également dissoudre les deux chambres du parlement. Il peut s'adresser des messages à la nation ainsi qu'au parlement. Il est le chef suprême des forces armées et nomme à ce titre aux emplois militaires. Il dispose d'un pouvoir 
exceptionnel consacré par l'article 59 : lorsque, par exemple, l'intégrité du territoire est menacée (mais également lorsque des évènements entravent le fonctionnement régulier des pouvoirs publics), il peut proclamer l'état d'exception. Il est alors habilité à prendre toutes les mesures qui s'imposent pour défendre l'intégrité territoriale ou permettre que les institutions de la République puissent fonctionner normalement.

Cette prépondérance de l'autorité royale peut être regrettable: la réforme de 2011 aurait dû consacrer l'autonomie du gouvernement en précisant que ce dernier serait dans l'avenir nommé par un vote de la chambre des représentants.

\section{L'Egypte}

En Egypte, quelques remarques allant dans le même sens peuvent être également formulées. Le Président de la République reste omniprésent au sein de la Constitution de 2014. Plusieurs dispositions tendent à appuyer une telle idée. Il lui revient ainsi de convoquer les deux chambres en session annuelle comme de clore cette session. Le Président de la République possède une pleine place dans la procédure législative puisqu'il a la possibilité de proposer des lois. En outre, il a le droit d'opposer son veto à la promulgation d'une loi et demander, à ce titre, à la Chambre des députés d'en débattre une nouvelle fois. Il possède un pouvoir de nomination étendu. C'est ainsi que si la constitution lui fait obligation de nommer le Premier ministre au sein de la majorité arrivée en tête des élections de la chambre c'est à condition que le gouvernement qu'il a nommé de manière discrétionnaire auparavant n'a pas obtenu la confiance de la Chambre. Il peut nommer également un certain nombre de personnes à la chambre des représentants sans que leur nombre soit supérieur à $5 \%$.

Le texte fondamental donne également un rôle important au chef de l'Etat dans la détermination de la politique nationale puisqu'il lui revient d'élaborer la politique générale avec la «collaboration» du Gouvernement. Il a la possibilité de convoquer le Gouvernement à chaque fois qu'il le juge utile. En effet, l'article 148 dispose que "Le président de la République peut convoquer le Gouvernement pour débattre de questions importantes, et préside les réunions auxquelles il participe».

Un article semble permettre au chef de l'Etat d'écarter le gouvernent pour exercer luimême certains pouvoirs. En effet, l'article 147 dispose que "Le Président de la République peut dispenser le gouvernement de mener à bien ses tâches s'il obtient l'approbation de la majorité de la chambre des représentants ".

Le chef de l'Etat apparait en outre comme le chef des armées (cela est d'autant vrai actuellement que le Président EL SISI est un ancien militaire qui a déposé l'ancien président MORSI). A ce titre, c'est à lui qu'il revient de déclarer la guerre comme l'état d'urgence sous certaines réserves. Le chef de l'Etat peut aussi consulter directement le peuple par voie de referendum sur toute question concernant les intérêts supérieurs de l'Etat.

Le président semble par ailleurs pouvoir exercer une influence sur le pouvoir judiciaire ce qui interroge sur la question de la séparation des pouvoirs et sur l'indépendance du pouvoir judiciaire. La Constitution précise en effet que le parquet est dirigé par un procureur général nommé par décret du présidentiel sur proposition du Conseil supérieur de la magistrature. L'indépendance de la justice porte à discussion si l'on a à l'esprit le véritable jugement de groupe organisé à l'encontre des frères musulmans lors de l'arrivée au pouvoir du général EL SISI. 

l'Etat est également flagrante lorsque l'on lit le texte constitutionnel. Le Président de la République exerce le pouvoir exécutif et préside le Conseil des ministres, et est finalement le véritable chef de l'exécutif. La Constitution précise en ce sens que le Gouvernement veille à la mise en œuvre de la politique générale de l'État conformément aux orientations et aux options fixées par le Président de la République. En outre, si le gouvernement peut prendre des décisions par ordonnance dans les matières qui sont normalement du domaine de la loi, c'est après accord du Président de la République.

74 S'agissant de la politique étrangère, il revient au Président d'en déterminer le contenu et c'est lui qui signe et ratifie les traités. Il peut mettre fin, quand bon lui semble, aux fonctions du Premier ministre qu'il lui revient également de nommer. La Constitution dispose explicitement que le Premier ministre et les ministres sont responsables devant le Président de la République.

\section{où il a la possibilité de communiquer avec celui-ci par des messages. Il peut en outre en} prononcer la dissolution. Il peut aussi saisir le peuple par referendum. domaine. Il est le chef suprême des forces armées et préside à ce titre les conseils et comités supérieurs de la défense nationale. A l'instar d'autres constitutions issues des mouvements révolutionnaires dans la zone géographique, il possède des pouvoirs 
exceptionnels dans certaines situations. Lorsqu'un péril imminent menace les institutions de la République, la sécurité ou l'indépendance de la Nation ou l'intégrité de son territoire et que le fonctionnement régulier des pouvoirs constitutionnels est entravé, le Président de la République adopte les «mesures exigées par ces circonstances » après consultation officielle du premier ministre, des présidents des assemblées et du Conseil constitutionnel. Enfin, il possède l'initiative de la révision constitutionnelle.

\section{Conclusion}

Les constitutions postrévolutionnaires, dans le monde arabe, tendent indéniablement vers la modernisation. Elles traduisent la volonté de renforcer les droits fondamentaux tout en allant vers un meilleur équilibre des pouvoirs. Néanmoins, il semble que ce nouvel élan ne soit pas abouti. Des limites persistent nécessitant de futures réformes et, la pratique du pouvoir contribue aussi à définir les caractéristiques de ces nouveaux régimes politiques. La question du renouveau constitutionnel dans le monde arabe reste ainsi ouverte et risque bien de continuer à faire l'objet d'une actualité régulière.

\section{NOTES}

1. Il en est de même pour les organisations syndicales.

2. Montesquieu, De l'esprit des lois, Livre XI, chap. VI.

3. D'autres Etats sont concernés par cette tendance.

En Mauritanie, avec la réforme du 20 mars 2012, on assiste à un renforcement de la responsabilité gouvernementale devant le pouvoir législatif. En effet, des dispositions précisaient déjà que le premier ministre est, solidairement avec les ministres, responsable devant l'Assemblée nationale ; la mise en jeu de la responsabilité politique résulte de la question de confiance ou de la motion de censure. La réforme constitutionnelle vient rajouter qu'au plus tard un mois après la nomination du Gouvernement, le Premier ministre présente son programme devant l'Assemblée nationale et engage la responsabilité du Gouvernement sur ce programme. Le statut du parlement est aussi renforcé. Les sessions parlementaires peuvent aller aujourd'hui jusqu'à 4 mois, contre 2 mois antérieurement. En outre, l'Assemblée nationale possède un délai de 45 jours afin de se prononcer en première lecture sur le projet de loi de finances contre 30 jours auparavant. De plus la mission du parlement sur le budget de l'Etat est précisée dans la mesure où il est mentionné que celui-ci contrôle l'exécution du budget de l'Etat et des budgets annexes. Un état des dépenses doit lui être fourni à la fin de chaque semestre pour le semestre précédent. Les comptes définitifs d'un exercice sont déposés au cours de la session budgétaire de l'année suivante et approuvés par une loi.

L'exemple jordanien est révélateur également de cette recherche d'équilibre. C'est ainsi que la réforme constitutionnelle jordanienne de 2011 réduit les prérogatives du 
gouvernement au bénéfice du Parlement dans le sens d'un plus grand équilibre des pouvoirs. Par exemple, la capacité de décréter des lois provisoires pendant les vacances parlementaires a été réduite. La possibilité pour le gouvernement de dissoudre le Parlement sans démissionner a également été limitée.

\section{ABSTRACTS}

Since 2011, the Arab world is confronted to a social and legal - especially constitutionalmutation. In Morocco, Jordan, Egypt or Tunisia, deep constitutional reforms are emerging. These changes are reaction against previous regimes characterized by their authorities, concentration of powers and violation of human rights. Several questions will be then explored: what are the positive contributions of these constitutional movements? Can they be analyzed as revival of human rights? Do they exit in building a balance of powers? What about the scope of the new constitutional reforms?

Le monde arabe, depuis 2011, vit une mutation tant sociale que juridique et, plus précisément, constitutionnelle. En effet, que ce soit au Maroc, en Jordanie, en Egypte ou encore en Tunisie, des réformes constitutionnelles de grandes ampleurs ont pu voir le jour. Ces mutations profondes se veulent une réponse aux régimes qui prévalaient par le passé : des régimes autoritaires, à forte concentration du pouvoir, une séparation des pouvoirs relative et de nombreuses atteintes aux droits de l'homme. Il convient alors de se poser plusieurs questions : quels ont été les apports de ces mouvements constitutionnels ? Peut-on parler de renouveau des droits de l'homme? Assiste$\mathrm{t}$-on à une tentative de mise en place des conditions d'un équilibre des pouvoirs? Quid de la portée de ces nouvelles réformes constitutionnelles? On envisagera les réponses apportées les principales nouvelles constitutions.

\section{INDEX}

Mots-clés: Constitution - Droits de l'homme - Séparation des pouvoirs - Mutations constitutionnelles - Démocratie

Keywords: Constitution - Human rights - Separation of power - Constitutional transition Democracy

\section{AUTHOR}

\section{MALIK BOUMÉDIENE}

Malik Boumediene est Maître de conférences, Habilité à Diriger les Recherches à l'université de Toulouse Jean Jaurès. Il est membre du Centre de Recherche et d'Etude sur les droits fondamentaux (Université Paris 10). Il travaille sur la question de la démocratie, des droits de l'homme, des transitions constitutionnelles. 